\title{
Application of co-expressed genes to articular cartilage: New hope for the treatment of osteoarthritis (Review)
}

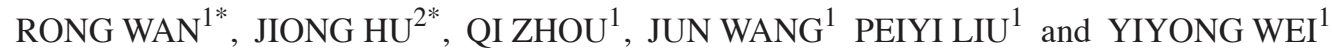 \\ ${ }^{1}$ Shanghai Key Laboratory for Prevention and Treatment of Bone and Joint Diseases with Integrated Chinese- \\ Western Medicine, Shanghai Institute of Traumatology and Orthopaedics, Ruijin Hospital, Shanghai Jiaotong \\ University School of Medicine, Shanghai 200025; ${ }^{2}$ Department of Orthopaedics, The Second Affiliated \\ Hospital of Zhejiang Traditional Chinese Medicine University, Hangzhou, Zhejiang 310005, P.R. China
}

Received August 2, 2011; Accepted October 21, 2011

DOI: $10.3892 / \mathrm{mmr} .2012 .859$

\begin{abstract}
Osteoarthritis (OA), with a high prevalence and economic impact, is a progressive diarthrodial joint disease that substantially reduces quality of life and is mainly characterized by degradation of the extracellular matrix (ECM) and the loss of a chondrogenic phenotype in articular cartilage. Strategic targeting of therapeutic genes to OA cartilage may offer potent alternatives for restoring the structure of the damaged cartilage. $\alpha 2$-macroglobulin $(\alpha 2 \mathrm{M})$, a member of the $\alpha 2 \mathrm{M}$ family of proteins, prevents the degradation of the ECM by inhibiting the activity of a disintegrin-metalloproteinases with thrombospondin motifs (ADAMTSs) and matrix metalloproteinases (MMPs). Sox9, a key chondrogenic transcription factor, plays a crucial role in the development and maintenance of the chondrogenic phenotype. Therefore, modulation of the OA cartilage by genetically modifying the levels of $\alpha 2 \mathrm{M}$ and Sox 9 expression may be advantageous in ameliorating the course of OA. To acquire long-lasting expression of the $\alpha 2 \mathrm{M}$ and Sox 9 genes, gene transfer systems are required. The chitosan vector system is expected to be useful for direct gene therapy for joint disease. Thus, we conclude that co-expression of the $\alpha 2 \mathrm{M}$ and Sox 9 genes, combined with chitosan-mediated gene delivery, will offer potential as a novel means by which to treat OA via intra-articular injection.
\end{abstract}

\section{Contents}

1. Osteoarthritic characteristics

Correspondence to: Dr Yiyong Wei, Key Laboratory of Science and Technology Commission, Department of Orthopaedics, Institute of T and O, Ruijin Hospital, Shanghai Jiaotong University School of Medicine, Shanghai 200025, P.R. China

E-mail: dr.yiyongwei@yahoo.com

*Contributed equally

Key words: osteoarthritis, articular cartilage, Sox9, chitosan-mediated gene delivery, $\alpha 2$-macroglobulin
2. Damage of cartilage matrix and $\alpha 2$-macroglobulin

3. Loss of chondrogenic phenotype and Sox 9

4. Gene therapy of osteoarthritis

5. Future directions

\section{Osteoarthritic characteristics}

Osteoarthritis (OA) is a degenerative joint disease that significantly impacts quality of life. Numerous factors varying from mechanical disruption to gene mutations in structural proteins have been proposed as contributors to the development of OA. The progression of the disease is slow and a frequent end point is destruction of the articular cartilage, which leads to limitation of joint movement, joint deformity, inflammation and severe pain (1). The pathological characteristics of OA are predominantly involved in the degradation of the extracellular matrix (ECM) and loss of a chondrogenic phenotype in cartilage.

\section{Damage of cartilage matrix and $\alpha 2$-macroglobulin}

Among the mechanisms responsible for OA cartilage matrix damage, disintegrin-metalloproteinases with thrombospondin motifs (ADAMTSs) and the matrix metalloproteinases (MMPs) have been indentified as being of key importance (Fig. 1). ADAMTSs are the main proteinases responsible for the cleavage of aggrecan and cartilage oligomeric matrix protein (COMP, a prominent noncollagenous component of cartilage) in the early event of OA. Later, MMPs participate in the process and continue with the degradation of collagen (2). A number of the ADAMTS protein family, including ADAMTS-1, $-4,-5,-7$ and -12 , play a crucial role in the process of OA. The study demonstrated that ADAMTS-4 and ADAMTS-5 had intensive aggrecan-degradation activity compared to other aggrecanases (ADAMTS-1) in vitro and were currently recognized as the most active aggrecanases in vivo $(3,4)$. In addition to aggrecan, ADAMTS-4 was also shown to cleave COMP (5). A recent study demonstrated that ADAMTS-7 and -12 were also able to digest COMP in vitro, and that their levels were significantly upregulated in OA (6). The MMPs consist of a number of subsets of enzymes 


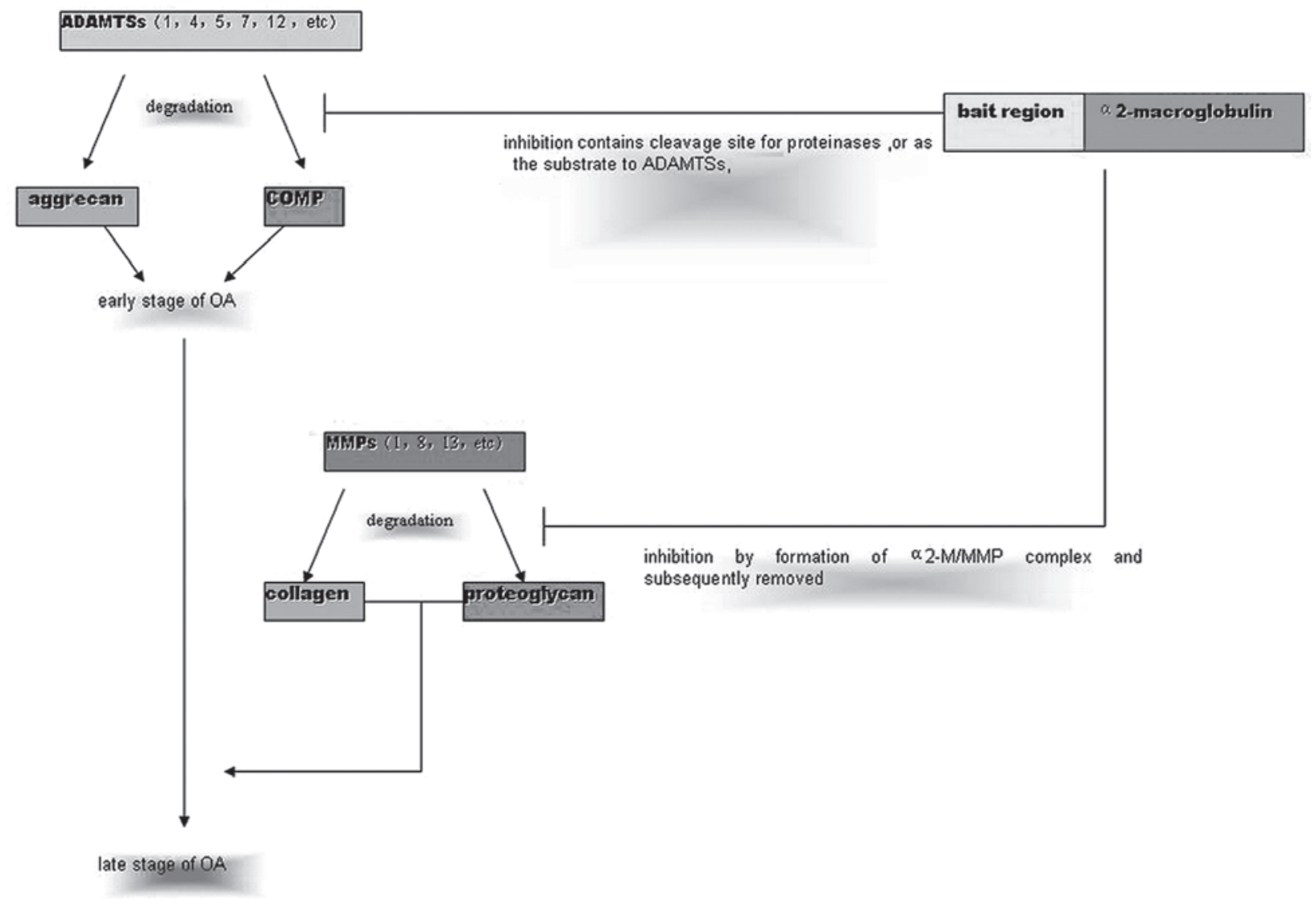

Figure 1. Disintegrin-metalloproteinases with thrombospondin motifs (ADAMTSs), the matrix metalloproteinases (MMPs), $\alpha 2$-macroglobulin ( $\alpha 2 \mathrm{M})$ and osteoarthritis. OA, osteoarthritis; COMP, cartilage oligomeric matrix protein.

including collagenases, gelatinases, stromelysins, matrilysins and the membrane-type MMPs. The most significant members of the MMP family with respect to OA are the collagenases (MMP-1, -8 and -13). Of the collagenases, MMP-13 prefers to cleave type II collagen while MMP-1 and MMP-8 prefer to cleave types III and I. In addition to collagen, MMP-13 also degrades the proteoglycan molecule, aggrecan, giving it a dual role in matrix destruction $(7,8)$.

Therapies aimed at inhibiting the activity of ADAMTSs and MMPs may slow progressive cartilage erosion and delay or even prevent the development of end-stage disease. $\alpha 2$-macroglobulin $(\alpha 2 \mathrm{M})$ is a member of the $\alpha 2 \mathrm{M}$ family of proteins that circulate in the plasma at relatively high levels (2-4 $\mathrm{mg} / \mathrm{ml})$, and is also found in the joint fluid at similar concentrations (9). Since each subunit of the $\alpha \mathrm{M}$ molecule has a region referred to as the 'bait region', which contains a cleavage site for a variety of proteinases (10), $\alpha 2 \mathrm{M}$ is known as a ubiquitous proteinase inhibitor. An earlier study suggested that ADAMTS-1 activity may be inhibited by $\alpha 2 \mathrm{M}$ and that inhibition of the aggrecanase may be mediated by proteolysis of the bait region within $\alpha 2 \mathrm{M}$ (11). More recent studies have demonstrated that $\alpha 2 \mathrm{M}$ is a novel substrate for ADAMTS-4, -5, -7 and -12, and may inhibit their activity in a concentration-dependent manner $(6,10)$. In addition, studies have also demonstrated that $\alpha 2 \mathrm{M}$ may be capable of inhibiting activated MMPs such as MMP-13 in body fluids $(12,13)$. The studies suggested that the activated MMPs were entrapped by $\alpha 2 \mathrm{M}$ and formed a complex with $\alpha 2 \mathrm{M}$ in its presence, that the active site of the MMPs in the $\alpha 2 \mathrm{M} / \mathrm{MMP}$ complex was shielded and that the complex was also rapidly cleared by the body (13).

\section{Loss of chondrogenic phenotype and Sox9}

In addition to the degradation of the ECM, the loss of a chondrogenic phenotype is also a significant characteristic in OA. Chondrocytes are the only cell type found within articular cartilage and they are embedded in the tissue's highly specialized ECM. The interaction of chondrocytes with ECM makes a crucial contribution to the maintenance of a chondrogenic phenotype (14). Therefore, breakdown of ECM components may affect the homeostasis of chondrocytes and lead to the loss of a chondrogenic phenotype, which would further accelerate the pathological course of OA.

Sox 9, a key chondrogenic transcription factor, played a significant role in the development and maintenance of the chondrogenic phenotype (15). One study demonstrated that Sox 9 expression was relatively high in normal cartilage but that its transcript levels were substantially reduced in OA accompanied with the degradation of the ECM, this study also suggested that a reduction of Sox 9 transcript levels in osteoarthritic chondrocytes may be responsible for the loss of phenotypic stability of osteoarthritic chondrocytes $(16,17)$. This was further supported by the fact that transduction with 
Sox 9 enhanced re-expression of the chondrogenic phenotype in osteoarthritic chondrocytes (18). Thus, upregulation of Sox9 expression may promote the recovery of a chondrogenic phenotype.

\section{Gene therapy of osteoarthritis}

Genetic engineering offers a new treatment approach to the destructive processes in OA (19). Various efficacious genes have been found, and numerous genes have been induced into synovial cells (synoviocites) and modified so that the destructive processes of OA can be delayed or prevented. A recent study has suggested that adenovirus-mediated kallistatin gene transfer ameliorates disease progression in a rat model of OA (20). Gelse et al have also demonstrated that autologous periosteal cells stimulated ex vivo by bone morphogenetic protein 2 (BMP-2) gene transfer (adeno-associated virus and adenovirus vectors) can resurface large partial-thickness cartilage defects in a porcine model of OA (21). In addition, Zhang et al have shown that chitosan-DNA nanoparticles containing interleukin-1-receptor antagonist (IL-1Ra) are capable of reducing the severity of cartilage lesions by injecting directly into the knee joint cavities of OA rabbits (22). Therefore, gene transfers of defined genes encoding therapeutic proteins represent a promising way to efficiently treat OA.

\section{Future directions}

Due to the complex pathology of OA, gene transfer strategies based on co-expression of $\alpha 2 \mathrm{M}$ and Sox 9 genes will become of major interest for interfering with key process of the disease, and this will also be a highly specific targeting of OA-relevant mechanisms. At present, there are primarily two types of gene transfer system: viral and non-viral vectors. Although viral vectors acquired some benefits, non-viral vector delivery systems for gene therapy have been increasingly proposed as safer alternatives to viral vectors and they provide nontoxicity, low immunogenicity, stability, high DNA binding efficiency and easy manufacture. As a type of non-viral gene delivery system, chitosan has a host of other advantages: i) protecting DNA from nuclease degradation; ii) enhancing the transport of DNA across the cell membrane through interaction with specific membrane receptors; iii) releasing-gene in a controlled manner. In addition, due to the analogous structure in chitosan with glycosaminoglycans - one of the components of cartilage-specific ECM - chitosan has also been used in the treatment of certain joint diseases. Therefore, we propose that intra-articular injection of chitosan/plasmid DNA (containing $\alpha 2 \mathrm{M}$ and Sox9 genes cloned into a plasmid as a whole body) will offer potential as a novel means to ameliorate OA.

\section{Acknowledgements}

This work was supported by the National Natural Science Foundation of China (No. 30801164) and the Innovation Program of Shanghai Municipal Education Commission (No. 09YZ107).

\section{References}

1. Goldberg VM, Buckwalter J, Halpin M, et al: Recommendations of the OARSI FDA Osteoarthritis Devices Working Group. Osteoarthritis Cartilage 19: 509-514, 2011.

2. Rengel Y, Ospelt $\mathrm{C}$ and Gay S: Proteinases in the joint: Clinical relevance of proteinases in joint destruction. Arthritis Res Ther 9: 221, 2007.

3. Glasson SS, Askew R, Sheppard B, et al: Deletion of active ADAMTS5 prevents cartilage degradation in a murine model of osteoarthritis. Nature 434: 644-648, 2005.

4. Fosang AJ, Rogerson FM, East CJ and Stanton H: ADAMTS-5: the story so far. Eur Cell Mater 15: 11-26, 2008.

5. Malfait AM, Liu RQ, Ijiri K, Komiya S and Tortorella MD: Inhibition of ADAM-TS4 and ADAM-TS5 prevents aggrecan degradation in osteoarthritic cartilage. J Biol Chem 277: 22201-22208, 2002.

6. Luan Y, Kong L, Howell DR, et al: Inhibition of ADAMTS-7 and ADAMTS-12 degradation of cartilage oligomeric matrix protein by $\alpha-2$-macroglobulin. Osteoarthritis Cartilage 16: 1413-1420, 2008.

7. Burrage PS, Mix KS and Brinckerhoff CE: Matrix metalloproteinases: Role in arthritis. Front Biosci 11: 529-543, 2006.

8. Brighton CT, Wang W and Clark CC: The effect of electrical fields on gene and protein expression in human osteoarthritic cartilage explants. J Bone Joint Surg Am 90: 833-848, 2008.

9. Chu CT, Howard GC, Misra UK and Pizzo SV: $\alpha 2$-macroglobulin: a sensor for proteolysis. Ann NY Acad Sci 737: 291-307, 1994.

10. Tortorella MD, Arner EC, Hills R, et al: $\alpha 2$-macroglobulin is a novel substrate for ADAMTS-4 and ADAMTS-5 and represents an endogenous inhibitor of these enzymes. J Biol Chem 279: 17554-17561, 2004.

11. Kuno K, Terashima $\mathrm{Y}$ and Matsushima K: ADAMTS-1 is an active metalloproteinase associated with the extracellular matrix. J Biol Chem 274: 18821-18826, 1999.

12. Beekman B, Drijfhout JW, Ronday HK and TeKoppele JM: Fluorogenic MMP activity assay for plasma including MMPs complexed to $\alpha 2$-macroglobulin. Ann NY Acad Sci 878: $150-158,1999$.

13. Tchetverikov I, Verzijl N, Huizinga TW, TeKoppele JM, Hanemaaijer R and DeGroot J: Active MMPs captured by a2 macroglobulin as a marker of disease activity in rheumatoid arthritis. Clin Exp Rheumatol 21: 711-718, 2003.

14. Hardingham TE, Oldershaw RA and Tew SR: Cartilage, SOX9 and Notch signals in chondrogenesis. J Anat 209: 469-480, 2006.

15. Haag J, Gebhard PM and Aigner T: SOX gene expression in human osteoarthritic cartilage. Pathobiology 75: 195-199, 2008.

16. Tew SR, Li Y, Pothacharoen P, Tweats LM, Hawkins RE and Hardingham TE: Retroviral transduction with SOX9 enhances re-expression of the chondrocyte phenotype in passaged osteoarthritic human articular chondrocytes. Osteoarthritis Cartilage 13: 80-89, 2005.

17. Yang Z, Huang CY, Candiotti KA, et al: Sox-9 facilitates differentiation of adipose tissue-derived stem cells into a chondrocyte-like phenotype in vitro. J Orthop Res 29: 1291-1297, 2011.

18. Lee JS and Im GI: SOX trio decrease in the articular cartilage with the advancement of osteoarthritis. Connect Tissue Res: July 5, 2011 (Epub ahead of print).

19. Kofron MD and Laurencin CT: Orthopaedic applications of gene therapy. Curr Gene Ther 5: 37-61, 2005.

20. Hsieh JL, Shen PC, Shiau AL, et al: Adenovirus-mediated kallistatin gene transfer ameliorates disease progression in a rat model of osteoarthritis induced by anterior cruciate ligament transection. Hum Gene Ther 20: 147-158, 2009.

21. Gelse K, Muhle C, Franke O, et al: Cell-based resurfacing of large cartilage defects: long-term evaluation of grafts from autologous transgene-activated periosteal cells in a porcine model of osteoarthritis. Arthritis Rheum 58: 475-488, 2008.

22. Zhang X, Yu C, Xushi, Zhang C, Tang T and Dai K: Direct chitosan-mediated gene delivery to the rabbit knee joints in vitro and in vivo. Biochem Biophys Res Commun 341: 202-208, 2006. 